\title{
Photothermal bleaching and recovery analysis in photoacoustic microscopy
}

Chiye Li, Chi Zhang, Liang Gao, Alejandro GarciaUribe, Lihong V. Wang

Chiye Li, Chi Zhang, Liang Gao, Alejandro Garcia-Uribe, Lihong V. Wang, "Photothermal bleaching and recovery analysis in photoacoustic microscopy," Proc. SPIE 8943, Photons Plus Ultrasound: Imaging and Sensing 2014, 89435Q (3 March 2014); doi: 10.1117/12.2040936

SPIE. Event: SPIE BiOS, 2014, San Francisco, California, United States 


\title{
Photothermal bleaching and recovery analysis in photoacoustic microscopy
}

\author{
Chiye Li, Chi Zhang, Liang Gao, Alejandro Garcia-Uribe, and Lihong V. Wang * \\ Optical Imaging Laboratory, Department of Biomedical Engineering, \\ Washington University in St. Louis, One Brookings Drive, Campus Box 1097, \\ St. Louis, Missouri, 63130, USA
}

\begin{abstract}
A novel method - photoacoustic recovery after photothermal bleaching (PRAP) - is proposed and implemented to study particle dynamics and medium properties at the micron scale via photoacoustic imaging. PRAP is an intuitive way to visualize as well as quantify dynamic processes in many kinds of media. We demonstrate PRAP first in a phantom study, and then in live cells. PRAP provides high signal-to-noise ratio imaging with minimal bleaching-induced artifacts during the recovery stage, ideal for monitoring the diffusive and kinetic phenomena inside a cell.
\end{abstract}

Keywords: Photothermal bleaching, nanoparticle, diffusion, kinetics, photoacoustic microscopy.

\section{INTRODUCTION}

Fluorescence recovery after photobleaching (FRAP) is a widespread method for investigating molecule dynamics in cells. In FRAP, fluorophores within a region of interest are first photobleached by a high-intensity laser, and then the diffusion of new fluorophores into this bleached region is monitored with a low-intensity excitation over a period of time ${ }^{1}$. By analyzing the temporal profile of the fluorescence signal, the properties of molecules and their surrounding media can be revealed ${ }^{2}$.

In photoacoustic microscopy (PAM) ${ }^{3}$, photoacoustic recovery after photothermal bleaching (PRAP) can similarly provide dynamic information, but is different from FRAP in some aspects. First, the photothermal bleaching of gold nanoparticles (GNPs) in PAM behaves differently before and after these absorbers are raised to a critical temperature by the excitation laser pulses ${ }^{4}$. The nanoparticles can be easily photothermally bleached with a relatively high intensity laser, and readily monitored with negligible bleaching at the stage of recovery using sub-threshold laser pulse energy. Second, the photothermal bleaching of gold nanoparticle includes only non-reversible deformation and evaporation with no toxin generated. Therefore, PRAP won't affect the normal cellular events significantly, and makes a good technique for live cell imaging.

In this study, PRAP is demonstrated first in a phantom study, showing the solution viscosity change when glycerol is added to water. Then, PRAP is demonstrated measuring live cells loaded with gold nanoparticles, indicating the flowability of cytoplasm. Cell staining was performed after the photoacoustic imaging experiment to evaluate the cell viability, and the result shows acceptable cell viability.

\section{MATERIALS AND METHODS}

\subsection{PRAP of gold nanoparticles in solutions}

The PRAP experiments were performed on a transmission-mode optical-resolution PAM system ${ }^{5}$. Colloidal gold nanoparticles with a diameter of $70 \mathrm{~nm}(83110-70$, TedPella) were mixed with deionized water and glycerol, and then

*Corresponding author: lhwang@wustl.edu

Photons Plus Ultrasound: Imaging and Sensing 2014, edited by Alexander A. Oraevsky, Lihong V. Wang, Proc. of SPIE Vol. 8943, 89435Q - (C) 2014 SPIE · CCC code: 1605-7422/14/\$18 - doi: 10.1117/12.2040936 
placed on a vortex to get solutions with uniform viscosity. Two solution media were used: deionized water and $30 \%$ glycerol aqueous solution. Both solutions had the same particle concentration of $120 \mathrm{pM}$. The solutions were then injected into a silicone tubing (300 $\mu \mathrm{m}$ inner diameter, 408060-002, VMR International). During this entire experiment, the laser beam was focused into the tubing and fixed at a stationary position (Figure 1(a)). First, the photoacoustic signal from the solution was measured as the baseline amplitude before bleaching by an attenuated laser beam $(\sim 200$ $\mathrm{nJ} /$ pulse). Then the full power of the laser ( $\sim 6000 \mathrm{~nJ} /$ pulse $)$ was utilized to bleach the gold nanoparticles. The bleaching duration was $2 \mathrm{~s}$. After bleaching, the light source was switched back to the attenuated beam to monitor the signal change over time.

\section{(a)}

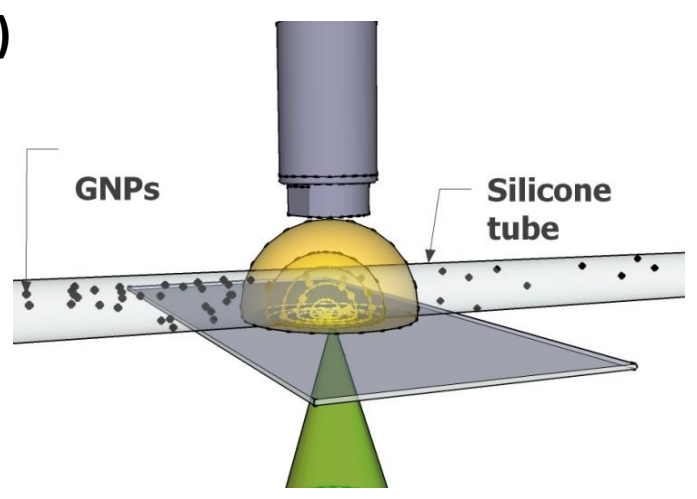

(b)

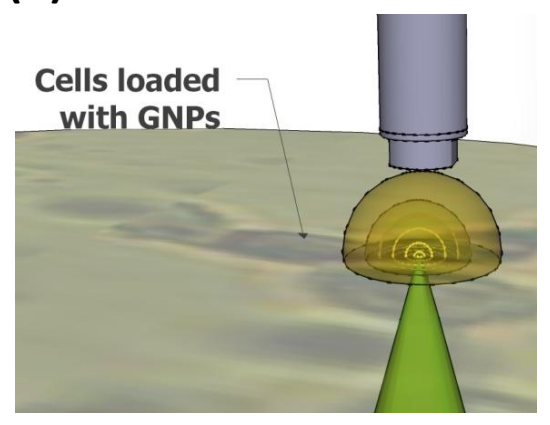

Figure 1. (a) Representation of the setup for the GNPs phantom experiment. The sample and ultrasound transducer were immersed in water. (b) Representation of the setup for the cell experiment. The cells grew on the bottom of a petri dish and were immersed in phenol red-free culture medium.

\subsection{PRAP of gold nanoparticles in living cells}

For the cell experiment, NIH 3T3 fibroblast cells were used. Fibroblast cells were maintained in Dulbecco's Modified Eagle Medium (DMEM) supplemented with 10\% fetal bovine serum, $2 \mathrm{mM}$ glutamine, 100 unites $/ \mathrm{ml}$ penicillin, and $100 \mu \mathrm{g} / \mathrm{ml}$ streptomycin at $37 \mathrm{C}^{\circ}$ in $5 \% \mathrm{CO}$. Cells were divided every $\sim 72$ hours. For subculture, they were dispersed in $0.25 \%$ EDTA-trypsin and then seeded at $2-4 \times 10^{4}$ cells $/ \mathrm{cm}^{2} .24$ hours after the cells were seeded to the bottom of culture dishes, $70 \mathrm{~nm}$ diameter gold colloid were added to the culture medium with a final particle concentration of 6 $\mathrm{pM}$. The cells were incubated with the particles for another 24 hours to let them uptake the nanoparticles. Before being imaged, the cells adhering to the bottom of culture dish were washed with fresh culture medium three times to remove particles that had not been uptaken. Then the culture medium was changed to phenol red-free DMEM to avoid the interference of phenol red in photoacoustic measurement while ensuring good cell viability during the experiment. The ultrasound transducer was directly immersed in the culture medium (Figure 1(b)). Photothermal bleaching and photoacoustic measurements were achieved by the same method in the phantom experiment, except that raster scanning was applied to acquire images of the cells.

After photothermal bleaching, cells were stained with LIVE/DEAD viability kit (L-3224, Life Technologies). The cell slides were first rinsed with warm phosphate buffered saline (PBS) and then stained by $2 \mu \mathrm{M}$ calcein AM and $4 \mu \mathrm{M}$ ethidium homodimer in PBS for 45 minutes. After staining, the cell slides were washed with PBS and imaged with a fluorescence microscope. Green fluorescence images were acquired with a 450-490 nm excitation filter and a 500-550 $\mathrm{nm}$ emission filter. Red fluorescence images were acquired with a 530-585 nm excitation filter and a 575-630 nm emission filter.

\section{RESULTS}

We validated PRAP first in a simple phantom study, and then in a more complex diffusion observation involving live cells. In the phantom study, recovery temporal profile was recorded by PAM with low-energy pulses, as shown in Fig. 2. Under the assumptions of uniform particle concentration in the infinite surrounding medium and negligible diffusion during photothermal bleaching, the photoacoustic (PA) signal recovery is governed by 


$$
P(t)=P_{\infty}-\left(P_{\infty}-P_{0}\right) \exp (-k t),
$$

where $P(t)$ denotes the time-lapsed PA signal, $P_{\infty}$ represents the asymptotic value when time $t$ tends to infinity, $P_{0}$ is the instantaneous PA amplitude observed immediately after photothermal bleaching, and $k$ is the PA recovery rate. Fitting the PA recovery profile yields $k$ values of $0.11 \mathrm{~s}^{-1}$ for deionized water and $0.073 \mathrm{~s}^{-1}$ for $30 \%$ glycerol aqueous solution.

(a)

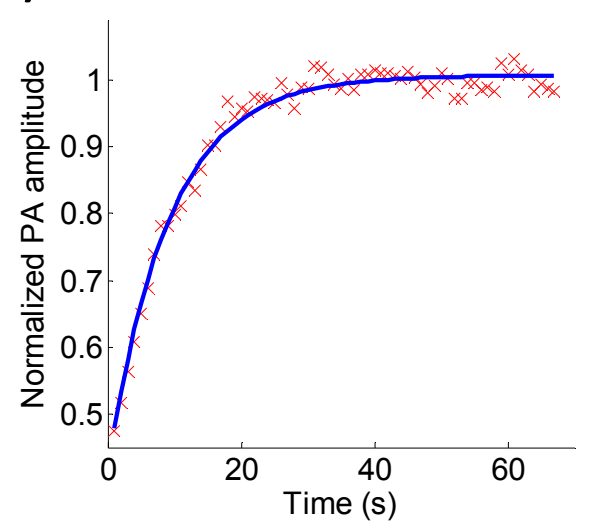

(b)

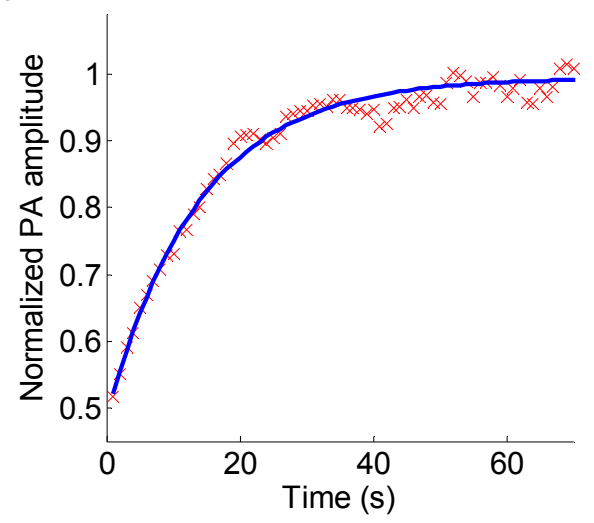

Figure 2. PA amplitude measured during recovery of $120 \mathrm{pM}, 70 \mathrm{~nm}$ colloidal GNPs in deionized water (a) and $30 \%$ glycerol aqueous solution (b).

To demonstrate PRAP in cell imaging applications, we loaded GNPs into NIH 3 T3 fibroblast cells and applied PRAP to visualize the intracellular particle diffusion. A region of interest was first chosen within a cell and photothermally bleached as shown in the Figure 3(a)(b). Then the PA signals from the bleached area were monitored after bleaching. The scan immediately after bleaching showed the signal decrease in the bleached area (Figure 3(b)) and the scan 30 minutes after bleaching showed the recovery of the PA signal (Figure 3(c)). The final PA signal did not recover to the initial value due to the conservation of unbleached particles within the cell.

\section{(a)}

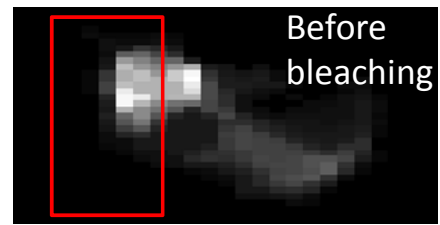

(b)

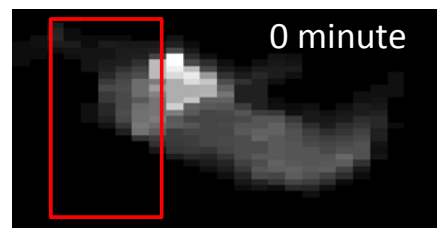

(c)
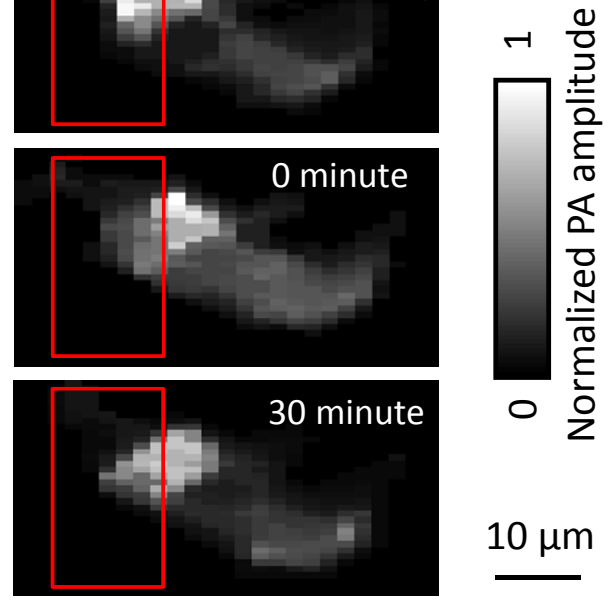

Figure 3. PRAP in a cell over time. Red blocks show the bleaching region. 
To evaluate PRAP's interruption to cell, viability test was performed. Cells in a square area were entirely bleached. The cell viability was tested by fluorescent staining. The results show that, with our experimental setup, about $50 \%$ of cells were still viable after the PRAP experiment (Figure 4).

(a)

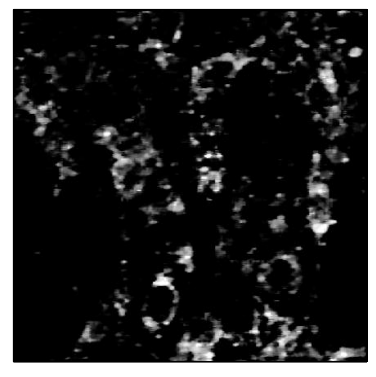

(c)

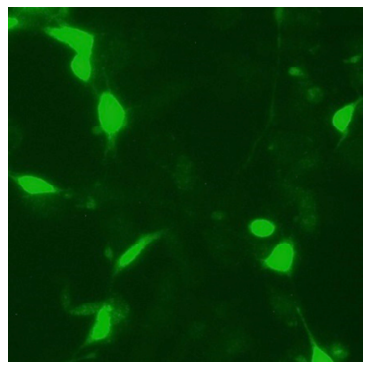

(b)

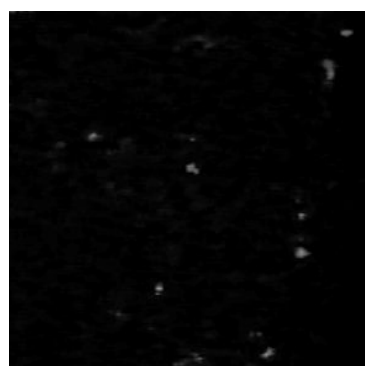

(d)

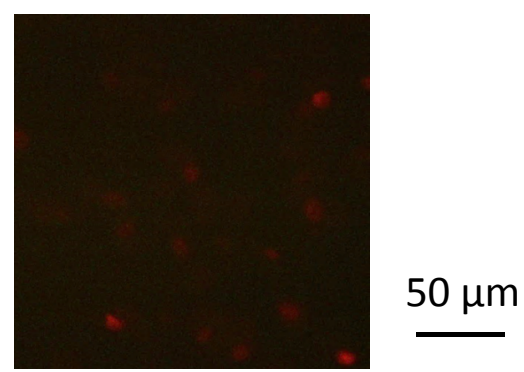

Figure 4. (a) PAM image of cells loaded with GNPs. (b) PAM images showing the same region as (a) was photothermally bleached. (c)(d) Live /dead fluorescent staining of the cells in the regions bleached photothermally. Live cells emit green fluorescence while dead cells emit red.

\section{DISCUSSION}

Gold nanoparticles were used as PA imaging contrast agents here because they offer superior chemical stability and are used extensively in live cell imaging ${ }^{6,7}$. In the phantom study, we measured the different recovery rates in different media. As expected, the PA recovery rate $k$ decreased when the medium viscosity increased. In the living cell experiment, we observed the recovery process. It is worth noting that the particle diffusion process in cell was relatively slow, attributed to the relatively large size of the bleached area. Additionally, the intracellular components form a filamentous meshwork, which restricts diffusion as a cytosolic sieve ${ }^{8,9}$. Moreover, the GNPs tend to aggregate upon cellular uptake ${ }^{10}$, which increases the particles' effective diameters and thereby slows down their diffusion. Depending on particle physicochemical properties (size, charge, coating, etc. ${ }^{11}$ and cellular biochemical conditions ${ }^{12}$, the formed cluster size varies. This variation in cluster size may impact the quantitative analysis of PRAP. To overcome this problem, the GNPs can be delivered into cells through microinjection ${ }^{13}$ instead of cellular endocytosis. Furthermore, when PRAP is performed outside of cells, such as on cell membranes, there is no aggregation, and therefore the analysis will not be affected.

\section{CONCLUSION}

In summary, we presented a new PA-imaging-based method, PRAP, for measuring intracellular diffusion rate. The experimental results demonstrated that PRAP has the capability to measure the mobility of particles in a viscous medium. In contrast to the conventional fluorescence-based approach, PRAP acquires high signal-to-noise ratio images with negligible bleaching-induced artifacts during PA recovery.

PRAP has the potential to facilitate nontoxic biological studies of diffusion at different scales. In the presented cellular experiment, GNPs were used to indicate the motion of cytoplasm. In the future, GNPs can be tagged to specific molecules or organelles via controlled labeling ${ }^{14}$, enabling studies of their intracellular diffusion. The performance of PRAP can be further improved by utilizing optimized PA contrast agents, such as silica-coated gold nanorods ${ }^{15}$, which 
exhibit smaller size, less disturbance to a living system, and higher PA excitation efficiency. Additionally, although not demonstrated here, PRAP can also be employed in biological tissues, allowing visualization and quantification of diffusive and kinetic processes at depths.

\section{ACKNOWLEDGEMENTS}

We thank Ms. Seema Dahlheimer for her attentive reading of the manuscript. This work was sponsored in part by National Institutes of Health grants DP1 EB016986 (NIH Director's Pioneer Award), R01 CA134539, and R01 CA159959. L.W. has a financial interest in Microphotoacoustics, Inc. and Endra, Inc., which, however, did not support this work. K.M. has a financial interest in Microphotoacoustics, Inc.

\section{REFERENCES}

[1] N. Klonis, M. Rug, I. Harper, M. Wickham, A. Cowman, and L. Tilley, "Fluorescence photobleaching analysis for the study of cellular dynamics," European Biophysics Journal, 31(1), 36-51 (2002).

[2] D. Axelrod, D. E. Koppel, J. Schlessinger, E. Elson, and W. W. Webb, "Mobility measurement by analysis of fluorescence photobleaching recovery kinetics," Biophysical Journal, 16(9), 1055-1069, (1976).

[3] L. V. Wang and S. Hu, "Photoacoustic tomography: in vivo imaging from organelles to organs," Science, 335, 1458-1462 (2012).

[4] L. Gao, L. Wang, C. Li, A. Garcia-Uribe, and L. V. Wang, "Photothermal bleaching in time-lapse photoacoustic microscopy," Journal of Biophotonics, 6, 1-6 (2012).

[5] C. Zhang, K. Maslov, and L. V. Wang, "Subwavelength-resolution label-free photoacoustic microscopy of optical absorption in vivo," Optics Letters, 35(19), 3195-3197 (2010).

[6] X. Yang, E. W. Stein, S. Ashkenazi, and L. V. Wang, "Nanoparticles for photoacoustic imaging," WIREs Nanomedicine and Nanobiotechnology, 1(4), 360-368 (2009).

[7] K. T. Thurn, E. Brown, A. Wu, S. Vogt, B. Lai, J. Maser, T. Paunesku, and G. E. Woloschak, "Nanoparticles for applications in cellular imaging," Nanoscale Research Letters, 2(9), 430-441 (2007).

[8] K. Luby-Phelps, D. L. Taylor, and F. Lanni, "Probing the structure of cytoplasm," Journal of Cell Biology, 102(6), 2015-2022 (1986).

[9] R. Swaminathan, S. Bicknese, N. Periasamy, and a S. Verkman, "Cytoplasmic viscosity near the cell plasma membrane: translational diffusion of a small fluorescent solute measured by total internal reflection-fluorescence photobleaching recovery," Biophysical Journal, 71(2), 1140-1151 (1996).

[10] S. Y. Nam, L. M. Ricles, L. J. Suggs, and S. Y. Emelianov, "Nonlinear photoacoustic signal increase from endocytosis of gold nanoparticles," Optics Letters, 37(22), 4708-4710 (2012).

[11] H. Y. Nam, S. M. Kwon, H. Chung, S.-Y. Lee, S.-H. Kwon, H. Jeon, Y. Kim, J. H. Park, J. Kim, S. Her, Y.-K. Oh, I. C. Kwon, K. Kim, and S. U. Jeong, "Cellular uptake mechanism and intracellular fate of hydrophobically modified glycol chitosan nanoparticles," Journal of Controlled Release, 135(3), 259-267, (2009).

[12] H.-H. Chen, C.-C. Chien, C. Petibois, C.-L. Wang, Y. S. Chu, S.-F. Lai, T.-E. Hua, Y.-Y. Chen, X. Cai, I. M. Kempson, Y. Hwu, and C. Margaritondo, "Quantitative analysis of nanoparticle internalization in mammalian cells by high resolution X-ray microscopy," Journal of Nanobiotechnology, 9(1), 14, (2011).

[13] R. Lévy, U. Shaheen, Y. Cesbron, and V. Sée, "Gold nanoparticles delivery in mammalian live cells: a critical review," Nano Reviews, 1, 1-18 (2010).

[14] H. Ba, J. Rodríguez-Fernández, F. D. Stefani, and J. Feldmann, "Immobilization of gold nanoparticles on living cell membranes upon controlled lipid binding," Nano Letters, 10(8), 3006-3012 (2010).

[15] Y.-S. Chen, W. Frey, S. Kim, P. Kruizinga, K. Homan, and S. Emelianov, "Silica-coated gold nanorods as photoacoustic signal nanoamplifiers," Nano Letters, 11(2), 348-354 (2011). 